\title{
SOAREX-8 Suborbital Experiments 2015 - A New Paradigm for Small Spacecraft Communication
}

\author{
Thom Stone \\ Computer Science Corp. \\ NASA Ames Research Center \\ Moffett Field, CA 94035 \\ Thom.Stone@nasa.gov
}

\author{
Marcus Murbach, Richard Alema, \\ Ray Gilstrap \\ NASA Ames Research Center \\ Moffett Field, CA 94035 \\ \{marcus.s.murbach, richard.l.alena, \\ ray.gilstrap\}@nasa.gov
}

\begin{abstract}
In 2015 NASA plans to launch a payload to $280 \mathrm{Km}$ altitude on a sounding rocket from the Wallops Flight Facility. This payload will contain several novel technologies that work together to demonstrate methodologies for space sample return missions and for nanosatellite communications in general. The payload will deploy and test an Exo-Brake, which slows the payload aerodynamically, providing eventual de-orbit and recovery of future ISS samples through a Small Payload Quick Return project. In addition, this flight addresses future Mars mission entry technology, space-to-space communications using the Iridium Short Messaging Service (SMS), GPS tracking, and wireless sensors using the ZigBee protocol. SOAREX-8 is being assembled and tested at Ames Research Center (ARC) and the NASA Engineering and Safety Center (NESC) is funding sensor and communications work. Open source Arduino technology and software are used for system control. The ZigBee modules used are XBee units that connect analog sensors for temperature, air pressure and acceleration measurement wirelessly to the payload telemetry system. Our team is developing methods for power distribution and module mounting, along with software for sensor integration, data assembly and downlink. We have demonstrated relaying telemetry to the ground using the Iridium satellite constellation on a previous flight, but the upcoming flight will be the first time we integrate useful flight test data from a ZigBee wireless sensor network. Wireless sensor data will measure the aerodynamic efficacy of the Exo-Brake permitting further onorbit flight tests of improved designs. The Exo-Brake is $5 \mathrm{~m}^{2}$ in area and will be stored in a container and deployed during ascent once the payload is jettisoned from the launch vehicle. We intend to further refine the hardware and continue testing on balloon launches, future sounding rocket flights and on nanosatellite missions. The use of standards-based and opensource hardware/software has allowed for this project to be completed with a very modest budget and a challenging schedule. There is a wealth of hardware and software available for both the Arduino platform and the XBee, all low-cost or open-source. Along with the Exo-Brake hardware and deployment discussion, this paper will describe in detail the system architecture emphasizing the successful use of opensource hardware and software to minimize effort and cost. Testing procedures, radio frequency interference (RFI) mitigation, success criteria and expected results will also be discussed. The use of Iridium short messaging capability for space-to-space links, standards-based wireless sensor networks, and other innovative communications technology are also presented.
\end{abstract}

\section{Table of Contents}

1. INTRODUCTION ...................................................1

2. SOAREX-8 OPERATIONAL DESCRIPTION ......2

U.S. Government work not protected by U.S. copyright
3. Wireless Sensor NetWork DeVELOPMENT3

4. FLIGHT QUALIFICATION ................................5

5. CONCLUSIONS AND FUTURE WORK..................5

ACKNOWLEDGEMENTS.....................................5

REFERENCES....................................................66

BIOGRAPHY ........................................................66

\section{INTRODUCTION}

In early 2015 a sounding rocket will loft the SOAREX-8 payload to $280 \mathrm{Km}$ above the Atlantic Ocean from the NASA Wallops Flight Facility (WFF) in Virginia. Upon reaching the ballistic apex, a single payload carrying several sets of experiments will be jettisoned. One of these located at the top of the payload 'box', the Exo-Brake, will unfold like Pegasus's wing. It will be a thin five-meter diameter gossamer metal film parachute that will spring from a small tube. The SOAREX-8 flight will deploy the first large ExoBrake (previous 1/10 scale Exo-brakes have been tested from the ISS through the TechEdSat nano-satellite series), which will stabilize the payload as it re-enters the atmosphere.

The SOAREX_8 Exo-Brake is a proof of concept for a method for sample return from the International Space Station (ISS) or as an option for landing small payloads on Mars. The project, based at NASA Ames Research Center (ARC) in Mountain View, California, is a low budget effort to flight test the Exo-Brake, among other goals. Using a small amount of funding from the NASA Engineering and Safety Center (NESC), the team is adding wireless instrumentation to help measure and understand the aerodynamics of the large-scale Exo-Brake. The authors and a group of interns under their direction are fabricating the hardware and software using low cost components. We have made extensive use of COTS open-source technology, saving considerable effort and cost.

One of the main drivers for this series of flight tests (SOAREX and the related orbital TechEdSat series) is prototyping technology that could be adapted for use with small satellites, especially those for education and research projects with modest budgets. Several of these technologies are mature and commercially available but are novel for use in spacecraft. This paper will describe selected interesting 
subsystems of this mission and how we made use of them. SOAREX-8 will demonstrate the use of:

- The large-scale Exo-Brake concept

- ZigBee wireless sensor networks

- Arduino processor boards

- Iridium Short Messaging Service (SMS) for telemetry relaying

- $\quad$ GPS tracking

\section{SOAREX-8 OPERATIONAL DESCRIPTION}

The sounding rocket program provides relatively easy access to space for small payloads. Sounding (as in ships measuring the depth of the sea or rivers) rockets are suborbital but can achieve perigee altitudes from 100 to $1500 \mathrm{~km}$. NASA launches sounding rockets from a variety of locations - which include Wallops Island, Virginia; White Sands, New Mexico; Poker Flat, Alaska; as well as a variety of international sites (e.g., Norway). These relatively low-cost solid rocket flights carry payloads that have major scientific value, prototypes for technology development, and university developed experiments. These rockets carry multiple payloads, and developers such as our team can take advantage of extra capacity to hitchhike to perform early flight tests in preparation for longer duration orbital experiments.

After the payload is jettisoned from the sounding rocket, a spring set will deploy the $5 \mathrm{~m}^{2}$ Exo-Brake. The Exo-Brake will be supported with a set of spokes and an inflation aid to rapidly deploy to the fully rigid state. The Exo-Brake is thus a 'parachute' designed to deploy and become rigid with very small dynamic pressure. At a sufficiently low ballistic coefficient $(\mathrm{M} / \mathrm{CdA}$; where $\mathrm{M}=$ mass, $\mathrm{Cd}=\mathrm{drag}$ coefficient, $\mathrm{A}=$ projected front surface area) of the order of $\sim 1 \mathrm{~kg} / \mathrm{m} 2$, payloads may be de-orbited from ISS orbital altitudes in less than 3 days.

In order to understand the physics of the deployment and operational behavior, a set of instrumented packages are being developed. An electronics package containing power, sensors, and Arduino boards with a ZigBee wireless transmitter is attached to the leeward apex of the Exo-brake. The sensors on the Exo-brake monitor static pressure and temperature, to be later compared to the analytical/numerical models being developed. Performing these kinds of measurements with traditional 'hard'-wired sensors would have been extremely complicated and would very likely compromise the deployment and erection of the Exo-Brake.

The figure below shows the Exo-Brake at $1 / 10$ scale as deployed from the TechEdSat-4 launch from International Space Station as an artist's illustration. The SOAREX-8 is a scaled up version of this with a similarly scaled 'satellite' forebody containing the electronic/communications systems.

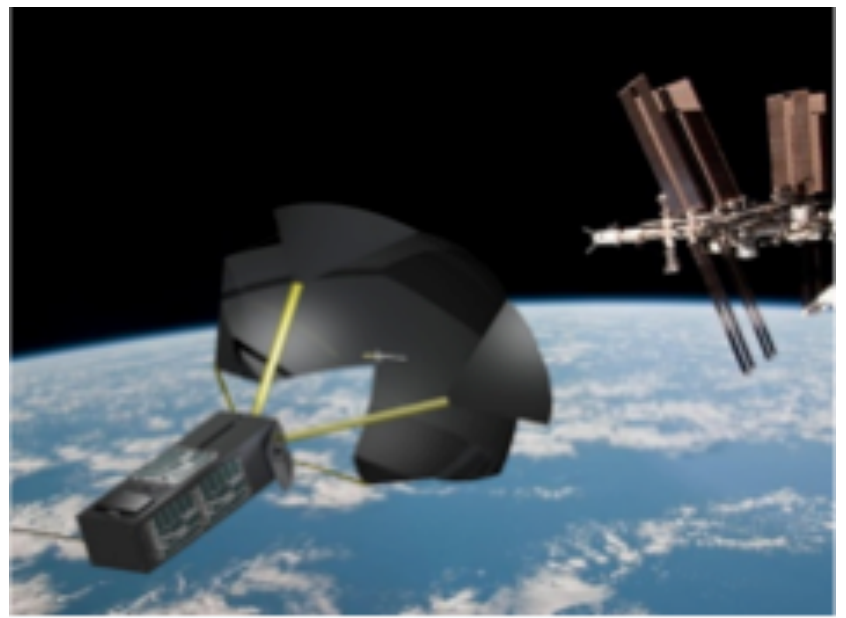

Figure 1. Exo-Brake as Deployed from the International Space Station

The 'nano-sat' payload deck (named thus as this configuration will be the core of future nano-satellites in the TechEdSat series) consists of a GPS receiver, additional ZigBee enabled sensors (static temperature/pressure and a 6 degree-of-freedom inertial measurement unit for measuring translational and rotational acceleration), a ZigBee coordinator and two Iridium transmitters all controlled by another Arduino. One of the Iridium modems is used for sending the GPS packets and the other for sending the full set of sensor outputs. Each Iridium unit will transmit one 140 byte packet every 10 seconds. Each packet will be received on the ground as an individual e-mail message from Iridium SMS via the Internet. The SOAREX-8 payload will splash down into the ocean and will not be recovered. The payload is shown in Figure 2, where the forward modular deck plates contain the different electronic modules for ease of integration/test.

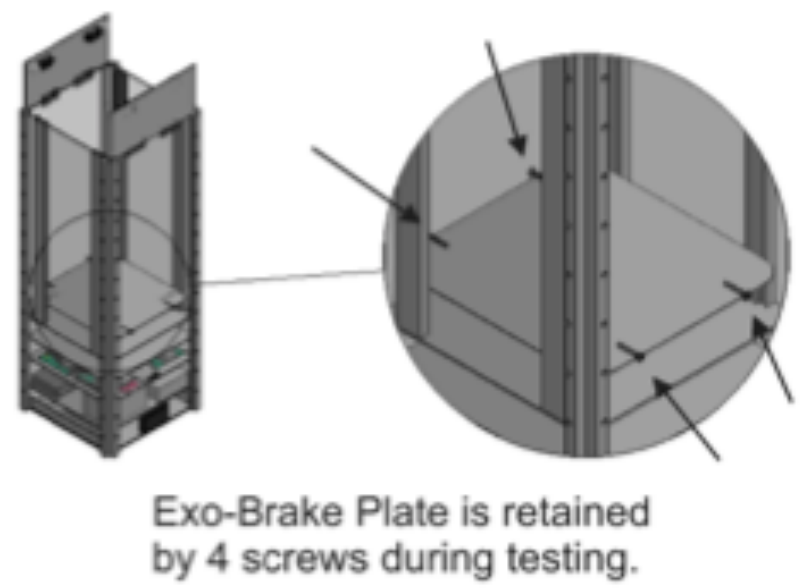

Figure 2. SOAREX-8 Payload Configuration 


\section{Wireless SenSOR NetWORK DEVElopMent}

The wireless sensor network (WSN) is an extension of the current SOAREX avionics system. The development cycle required producing hardware and software within about 3 months, so rapid prototyping techniques were used and much of the work was performed by interns at NASA Ames. The existing avionics system was based on an Arduino processor, which formats GPS packets into Iridium packets for downlink via Iridium SMS. It was critical for the success of this extension to build as much as possible on this flight verified foundation.

\section{Arduino Technology}

The concept for the Arduino open-source hardware was developed at the Interaction Design Institute Ivrea in Ivrea, Italy by Professor Massimo Banzi. The purpose was to build a simple, easy-to-use, low-cost processor board that students could use for projects. Professor Banzi further developed and commercialized it with a small group of engineers. The Arduino hardware and software are both completely opensource, with a rich set of libraries focused on input/output (I/O) functions.

The Arduino processor board consists of an Atmel ATmega2560 microprocessor and USB, serial, and analog ports. Various levels of processor performance are available. "Shield" (daughter) boards can be attached to extend functionality, and a large number of sensors are available for this platform. The great advantage of the board is in the open-source hardware and software developed for the platform and the large number of international users. Lights, mechanical devices, sensors, plumbing fixtures and everything imaginable have been interfaced to the Arduino platform. Arduino boards can control almost anything by modifying software available from open-source repositories, allowing prototypes to be built rapidly. The Arduino has been wedded to ZigBee modules for low bandwidth wireless communications between devices. For the SOAREX-8 we used ZigBee hardware from Digi International, who also provided help and advice for the project. Digi has a broad line of ZigBee modules called the XBee. We used the XBee shield attached to an Arduino Due for the remote sensor module, and an additional XBee attached to an Arduino Mega for the coordinator and payload interface. The project considered ZigBee technology from other vendors, but decided to stay within the Arduino ecosystem to maintain maximum compatibility with existing hardware and software.

The following diagram shows the system configuration for the WSN integrated with the Iridium unit. More details on the individual components are provided below.

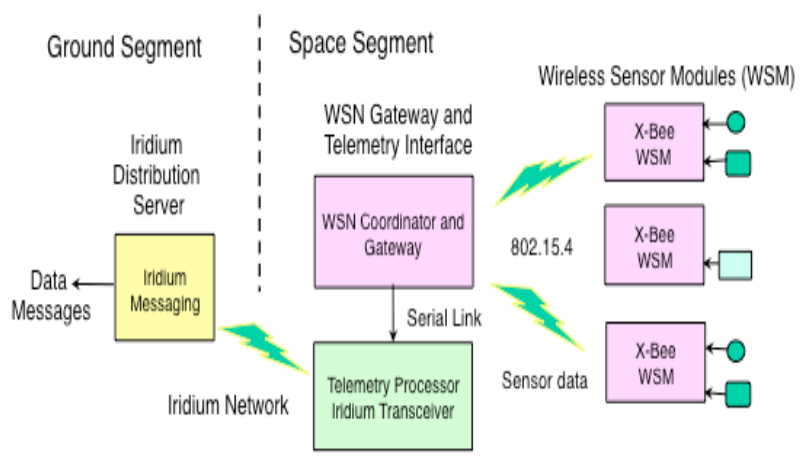

Figure 3. Wireless Sensor Network Diagram

\section{ZigBee Sensor Networks}

ZigBee is designed as a short-range wireless network standard. The design goals are low-power operation, use of the open Industrial Scientific and Medical (ISM) radio frequency bands (same as WiFi $802.11 \mathrm{~b}, \mathrm{~g}, \mathrm{n}$ ), and a layered protocol model. The IEEE 802.15.4 standard is used as the underlying physical layer, providing a self-organizing network. Like WiFi, ZigBee uses a "listen-before-talk," "back off on error" type of access protocol termed Carrier Sense Multi-Access (CSMA). The Personal Area Network (PAN) is based on the IEEE 802.15.4 standard describing the physical layer (PHY) and the media access control (MAC) mechanism. The ZigBee network layer resides above these and provides mesh routing, application layer support and implements the ZigBee framework for interoperable devices. It is a tiered architecture with end devices, routers, coordinators, and gateways. Routers can be used to extend range by repeating data transmissions and to provide redundancy via failover. ZigBee defines extensions supporting security and channel switching to avoid conflicts. The net data rate measures 104 kilobits per second-not high, but sufficient for sensor deployment. ZigBee uses Direct Sequence Spread Spectrum (DSSS) transmission, in which the ISM band is divided into 16 channels and each ZigBee network uses one channel. WiFi uses the same direct sequence technique but one $\mathrm{WiFi}$ channel is equivalent to four ZigBee channels.

ZigBee Standards are devised and enforced by the industryrun ZigBee Alliance. ZigBee is an evolving standard; the latest versions are ZigBee Pro, designed for large complex networks, and ZigBee 7 for small networks that do not require a rich feature set. The Alliance has hundreds of member companies, in three levels of membership: Promoter, Participant, and Adopter. So far, there are no government agencies involved in the ZigBee Alliance, which, aside from devising and approving the standards, provides for interoperability testing for all ZigBee devices.

ZigBee "profiles" are standard interoperation protocols and procedures developed for commercial sectors. Industry profiles describe the devices used, how they interact, and how they should be connected. Profiles allow for easy builds of complex networks. Profiles include Home Automation, Smart Energy and Medical device definitions. 
The ZigBee Alliance has collected a cluster library of higher-level building blocks for software. These can significantly reduce the effort to build new ZigBee applications.

In operation, the coordinator node forms the PAN by broadcasting a beacon requesting connections from routers and endpoints. The coordinator sets the RF channel and PAN ID and compliant nodes respond and join the PAN. The coordinator and routers maintain a dynamic routing table showing nearby PAN neighbors. Therefore they are able to directly send data across the PAN, or figure out how to do this by hopping nodes in the mesh network. The endpoints usually contain the sensors and can sleep for much of the time, greatly conserving power.

\section{Sensor Suite}

We are employing three types of sensors on SOAREX-8. We have mounted temperature and air pressure sensors directly on the Exo-Brake, connected to the Arduino/XBee and powered by a battery. Inside the body (see figure 1) are these same sensors and a six degree-of-freedom (6DOF) Inertial Measurement Unit (IMU). All are connected to the coordinator and Iridium downlink using XBee units. The software for running the XBees was developed in-house at ARC by staff and interns associated with the project team. Sensors were chosen based on the measurements needed and the environment encountered during payload reentry. The sensors need to survive vacuum, low temperatures (40C) and high temperatures in excess of $125 \mathrm{C}$.

The primary sensors are precision thermistors for temperature measurements and absolute pressure sensors for air pressure. The primary flight sensor module measures these properties in the lee of the Exo-brake, providing key aerodynamic data. A secondary sensor module measures these same quantities in the payload enclosure itself. The IMU is mounted on an additional sensor module in the enclosure and measures orientation along with translational and rotational velocity and acceleration. The coordinator samples each remote sensor module once every 10 seconds and forms a single Iridium packet with this data.

The SOAREX-8 flight system includes a GPS unit. The GPS uses a dedicated Iridium transmitter to track the reentry path to where the payload will hit the Atlantic, although we are not planning to retrieve it. The use of GPS for space vehicles required us to obtain a COCOM-unlocked unit. The GPS antennas are mounted to the outside of the payload enclosure to provide strong signals, and the GPS measurements are passed directly to the Arduino Mega through a serial data link.

\section{Iridium Communications}

One of us (M. Murbach) was able to coordinate testing of satellite-to-satellite links using the Iridium network for previous TechEdSat missions. ${ }^{1}$ SOAREX-8 will attempt to put this technology to practical use by sending key telemetry data to the ground during re-entry. Iridium consists of a constellation of 66 active satellites in polar orbits at $781 \mathrm{~km}$ above the earth and provides universal coverage to the earth's surface. The use of Iridium for space telemetry is novel. Iridium satellites maintain a backbone of intersatellite communications via Ka-band, so Internet connections can be maintained even as satellites come in and out of view. The system supports voice and Internet connections and emergency communications. One Iridium offering is the Short Messaging Service, a low cost service that allows for messages of up to 256 ASCII bytes to be forwarded. These messages can be received via e-mail. We found that the maximum throughput is one message every 10 seconds. Modems for SMS are small and make use of the AT command set derived from the old Hayes analog modems. Iridium can also send messages up to space using the same setup. This can allow for a command stream to small satellites. We intend to make use of this function for future missions, but currently only use the downlink.

\section{Software Functions}

A key advantage of the Arduino platform is the ease with which software can be developed for it. Using the freely available Arduino development environment and an existing library of XBee software functions, we were able to quickly develop software for both the primary Arduino Mega board and the XBee-based sensor boards.

The software running on the primary Mega board performs the key functions of the mission, deploying the Exo-Brake and coordinating communication of GPS and sensor data to Earth via the Iridium modems. Like all Arduino programs, the code performs initial setup once the board is powered, and then settles into a main loop that runs indefinitely. The initial setup phase includes basic housekeeping tasks such as initializing the mission clock and the control lines for the Iridium modem, and setting up serial communication with the XBee coordinator. The main loop first idles until the designated deployment time for the Exo-Brake, then triggers the Exo-Brake deployment actuators twice for redundancy. From that point, the loop continuously performs the following sequence of events:

- $\quad$ Check the Iridium receive memory buffer for any incoming commands that may have been transmitted to the satellite from the ground

- Wait up to three seconds for a packet of sensor data to arrive from the XBee coordinator

- Prepare an outbound packet containing the sensor data for transmission over the Iridium modem

- Initiate the Iridium transmission sequence and transmit the packet

- $\quad$ Listen for a response from the modem indicating that transmission has completed 
For the temperature and pressure sensor shields, the setup phase simply initializes communication with the XBee coordinator. The main loop continuously reads the raw analog voltages from the sensors, which are connected to analog input pins on the shield, converts the voltages into packets containing the corresponding digital values, and transmits the packets to the coordinator. A new packet is generated every three seconds. The 6DOF IMU shield code is almost identical except for additional initialization steps that are needed for the IMU during the setup phase.

There is a concurrent sampling loop running in the Arduino Mega, which hosts the GPS unit. The GPS is sampled every 5 seconds and a separate Iridium packet formed that just contains the GPS data. This packet is downlinked every 15 seconds as well.

\section{Flight Qualification}

Any system that operates in space must meet certain basic requirements associated with launch vehicle and payload safety. For example, our payload is launched in a powereddown state and activated only when above a certain altitude. A high-reliability altitude switch in a redundant configuration is used for activation and the payload supplies its own power from batteries. Moreover, since the payload system has to run over a wide range of air pressure and temperature, the prototype is subject to testing in a thermalvacuum chamber over the anticipated range of environmental conditions.

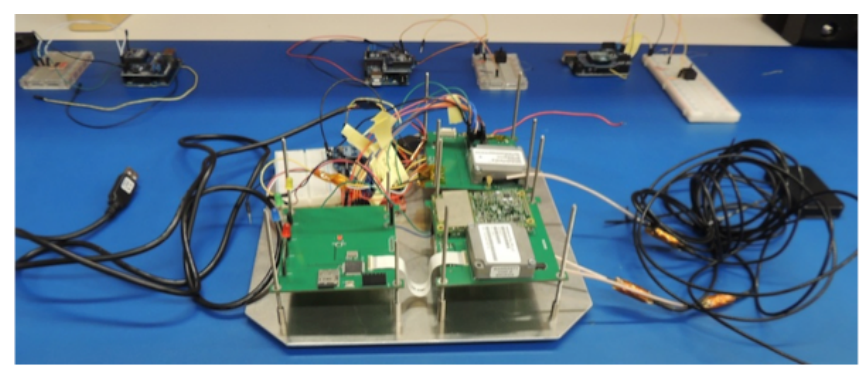

Figure 4. FlatSat Prototype

Our payload has operating transmitters and these must be certified for operation by conducting electromagnetic interference and compatibility testing (EMI/EMC). The output of the transmitters is measured, compared against EMI/EMC criteria and for compatibility with the Iridium transceiver and any other radio equipment on-board.

Lastly, mechanical integration and integrity must meet the shock and vibration requirements associated with rocket launch. The structures are evaluated for strength and durability.

\section{CONCLUSIONS AND FUTURE WORK}

The team was able to create a prototype WSN integrated with the SOAREX-8 payload system within 3 months of inception. This was possible by using the rapid prototyping capability provided by the Arduino and XBee product lines.
The hardware was readily available at low-cost and very short delivery times. The use of existing software libraries greatly simplified this key portion of the work. This work provided an excellent learning opportunity for our interns and even the senior members of the team. The project is proceeding to flight unit fabrication and flight qualification testing within the next few months.

This is an ongoing project. We intend to continue our rapid prototyping of wireless sensor networks and associated communication links. We are already planning more flights using high altitude balloons, additional sounding rocket flights, and the ISS small satellite facility for TechEdSat launches. The University of Idaho is our partner for high altitude balloon flights. TechEdSat4, our previous technology showcase, was just launched from Space Station in September 2014.

We are already designing the second generation of our wireless sensor network using less power and with a smaller footprint. We will be adding additional types of sensors and are thinking about using intelligent ZigBee modules to create smart spacecraft. We will be researching new power options such as super capacitors. We are attempting to implement a network using a formation of small and nanosatellites using ZigBee. We are experimenting with alternate computing options such as the Intel Edison board that will provide much greater processing and memory capacity for high-resolution video imaging. For communications, we will investigate and implement other space-to-space commercial options including commercial Ka-band offerings and extending the cellular network.

\section{ACKNOWLEDGeMents}

We would like to especially thank Gabriel Pearhill (University of Idaho) and Ryan Rivas (University of California, Riverside) for helping to lead the effort in code development and flight adaption. In addition, Ali Guarneros-Luna, Jose Mojica, Michael Scales, Kevin Sok, Thomas Shu, Anthony DiQuattro and Jose Ramil Seneris all contributed to the development of the overall payload. 


\section{REFERENCES}

[1] Murbach, Marcus S., Guarneros-Luna, Ali, Mojica, Jose, "TechEdSat-N : Pioneering The Use of the International Space Station as a Nano-Satellite Launch Platform," Cubesat Workshop, California Polytechnic State University, San Luis Obispo, March 23, 2014.

[2] Murbach, Marcus S., Boronowsky, Kenny M., Benton, Joshua E., White, Bruce, Fritzler, Erin, "Options for Returning Payloads from the ISS after the Termination of STS Flights, " 40th International Conference on Environmental Systems, Barcelona, AIAA 2010-6223, Spain, July 11-15, 2010.

[3] Faludi, Robert. Building Wireless Sensor Networks. Beijing: O'Reilly, 2010.

[4] Richard Alena,Thom Stone, Ray Gilstrap, Jarren Baldwin, and Pete Wilson. "Fault Tolerance in ZigBee Wireless Sensor Networks." IEEE Aerospace, 2010 .

[5] "Ubiquitous Sensor Networks (USN): ITU-T Technology Watch Briefing Report Series, No. 4." http://www.itu.int/dms_pub/itut/oth/23/01/T23010000040001PDFE.pdf

[6] Richard Alena, Fernando Figueroa, and John Ossenfort. "Intelligent Wireless Sensor Networks for Spacecraft Health Monitoring," AIAA Infotech Conference, 2012.

[7] "ZigBee Network Devices Overview." http://zigbee.org/Standards/ZigBeeNetworkDevices/Over view.aspx

[8] Thom Stone, Richard Alena, Jarren Baldwin, and Pete Wilson. "A Viable COTS Based Wireless Architecture for Spacecraft Avionics." IEEE Aerospace, 2012.

\section{BIOGRAPHY}

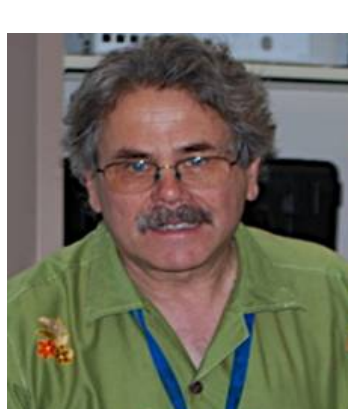

Thom Stone is a Senior Computer Scientist with Computer Sciences Corp. He is attached to the NASA Research and Engineering Network project at Ames Research Center (ARC). Mr. Stone has been at NASA ARC employed by various contractors since 1989. He was an engineer with the NASA Science Internet project office where he led the project that brought reliable Internet connections to remote locations including U.S. bases in Antarctica including McMurdo Station and Amundson Scott South Pole Station. He was principal engineer for communications for the NASA Search for Extraterrestrial Intelligence (SETI) project and was a senior engineer for the Space Station Biological Research Project. Before his involvement with NASA, Stone was employed in the computer and communications industry and taught telecommunications at the undergraduate level.

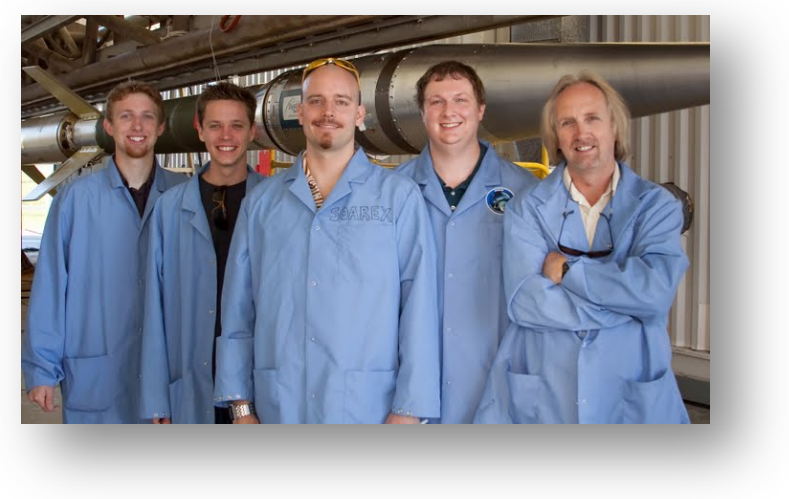

Marcus Murbach (on right) is the Principal Investigator of the SOAREX (Sub-Orbital Aerodynamic Re-entry Experiments) flight series, as well as the TechEdSat nano-satellite series. Besides the SOAREX-8 experiment, he is also working with the team on the TechEdSat-5 and -6 missions. His interests include hypersonics, satellite design, and extending the nano-satellite paradigm beyond Low Earth Orbit (LEO). He has worked at NASA Ames for 25 years and is also an Adjunct Professor at San Jose State University in the Aeronautics/Space Department. 


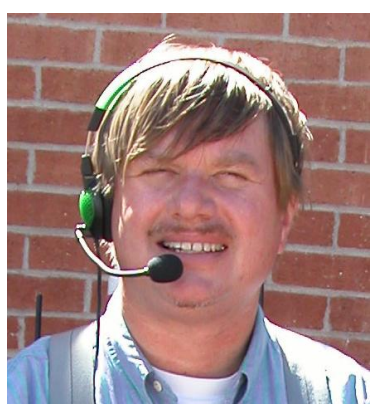

Richard L. Alena is a Computer Engineer in the Intelligent Systems Division at NASA Ames. Mr. Alena worked on the Ground Data System and performed Communications Analysis during operations for the LCROSS Lunar Mission and on avionics and software architectures for Lunar Surface Systems for human missions. He was the co-lead for the Advanced Diagnostic Systems for International Space Station (ISS) Project, developing model-based diagnostic tools for space operations. He was the chief architect of a flight experiment conducted aboard Shuttle and Mir using laptop computers, personal digital assistants and servers in a wireless network for the ISS. He was also the technical lead for the Databus Analysis Tool for International Space Station on-orbit diagnosis. He was group lead for Intelligent Mobile Technologies, developing planetary exploration systems for field simulations. Mr. Alena holds an M.S. in Electrical Engineering and Computer Science from the University of California, Berkeley. He is the winner of the NASA Silver Snoopy Award in 2002, a NASA Group Achievement Award in 1998 for his work on the ISS Phase 1 Program Team and a Space Flight Awareness Award in 1997.

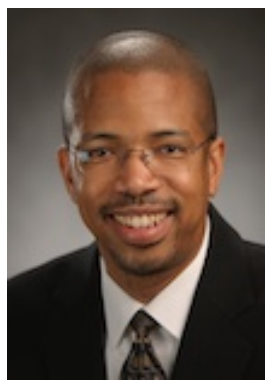

Ray Gilstrap is the Chief Technology Officer for Information Technology at NASA Ames Research Center in Moffett Field, California. $\mathrm{He}$ is responsible for identifying and evaluating cutting-edge information technologies for infusion into NASA missions and projects. With a background in computer networking, he has been involved in numerous projects in areas including high-performance networks, space communications, rapidly deployable satellite and wireless networking, computer security, and multimedia. He is a veteran of field science expeditions in remote desert and polar locations around the world. He holds a B.S. in Electrical Engineering from Florida A\&M University, and an M.S. in Electrical Engineering from the University of California, Berkeley. 